\title{
Opioid-induced proliferation of vascular endothelial cells
}

\author{
Sandra Leo ${ }^{1,2}$ \\ Rony Nuydens' \\ Theo F Meert ${ }^{\prime}$ \\ 'Pain and Neurology, CNS Department, \\ Johnson and Johnson Pharmaceutical \\ Research and Development, a division \\ of Janssen Pharmaceutica N.V, Beerse, \\ Belgium; ${ }^{2}$ Laboratory of Biological \\ Psychology, University of Leuven, \\ Leuven, Belgium
}

\begin{abstract}
Angiogenesis is an important issue in cancer research and opioids are often used to treat pain in cancer patients. Therefore it is important to know if the use of opioids is associated with an aberrant stimulation of tumor growth triggered by the stimulation of angiogenesis in cancer patients. Some studies in the literature have suggested the presence of the $\mu 3$ opioid receptor, known as the receptor for many opioids, on endothelial cells, which are key players in the process of angiogenesis. In this study we used endothelial cells known to express the $\mu 3$ opioid receptor (MOR3), to evaluate the effects of morphine on angiogenesis. We first investigated the effect of morphine on the proliferation of endothelial cells. We showed that morphine is able to stimulate vascular endothelial cell proliferation in vitro. This effect of morphine is mediated by the mitogen-activated protein kinase (MAPK) pathway as pre-treatment with PD98059 inhibited this excessive proliferation. Because previous studies indicated nitric oxide (NO) as a downstream messenger we investigated the role of NO in the aberrant proliferation of endothelial cells. Our data could not confirm these findings using intracellular NO measurements and quantitative fluorescence microscopy. The potential use and pitfalls of opioids in cancer patients is discussed in light of these negative findings.
\end{abstract}

Keywords: endothelial cells, morphine, cell proliferation, MAPK, nitric oxide, $\mu 3$ opioid receptor, angiogenesis

\section{Introduction}

Angiogenesis, the sprouting of new blood vessels, is fundamental for new organ development and for differentiation during embryogenesis, wound healing, and reproductive functions in adults. The vascular system is essential for supplying tissues with oxygen and nutrients and for the removal of metabolic end products. Angiogenesis is also an important factor in some pathological conditions, such as rheumatoid arthritis, proliferative retinopathies, and psoriasis, as well as tumor growth and metastasis. This complex process, during which endothelial cells play an important role, is tightly regulated by pro- and anti-angiogenic growth factors. Endothelial cells line the intimal surface of blood vessels, the barrier between blood and tissue. Angiogenesis proceeds in several steps including degradation of the basement membrane, migration of endothelial cells towards an angiogenic stimulus, proliferation of endothelial cells, lumen formation and the formation of a new extracellular matrix. Because of the prime role of endothelial cells during blood vessel formation, these cells are important targets in cancer research. ${ }^{1-3}$

For the treatment of pain in cancer patients, opioids are often used and morphine is an important example of such a pain-relieving drug. Opioid alkaloids, as well as endogenous opioid peptides, exert their analgesic effects on target tissues through the interaction with specific cell surface receptors including the $\delta$-, $\kappa$-, and $\mu$-opioid receptors. The $\mu$-opioid receptor (MOR) serves as the principle physiological target for most, clinically important, opioid analgesics including morphine and fentanyl. ${ }^{4}$ 
The opioid receptors are mainly localized in the nervous system, but it is already shown that endothelial cells also express a subtype on their cell surface. The $\mu_{3}$ opiate receptor is a high affinity, saturable opiate-binding site presumed to mediate morphine effects. So it is already demonstrated with endothelial cultures, that morphine affects endothelial cells possibly leading to vasodilatation. ${ }^{5}$ A clear mechanism for this effect was not put forward.

The $\mu_{3}$-opioid receptor is a G-protein coupled receptor (GPCR) that, after ligand binding, activates downstream G-proteins. ${ }^{4}$ This active G-protein can interact with other effector proteins and potentially stimulate different signal pathways, for example the mitogen-activated protein kinase (MAPK)-pathway. MAP-kinases are important intracellular components downstream of receptors and sensors and are able to split an extracellular stimulus to an intracellular response. This pathway exists in all eukaryotes and controls fundamental cellular processes such as proliferation, differentiation, survival and apoptosis. The basic arrangement includes a sensor (receptor) working upstream of a core module consisting of three kinases: a MAPK kinase kinase (MAPKKK) that phosphorylates and activates a MAPK kinase (MAPKK), which in turn activates MAPK. ${ }^{6}$ But also many receptors tyrosine kinases have been shown to activate the MAPK pathway. ${ }^{7}$ Previous studies have proposed nitric oxide (NO) as being the downstream mediator of the morphine effects. NO is a ubiquitously expressed mediator of intracellular signaling originating from various sources. We hypothesized that morphine may induce cell proliferation by activating MAPK via NO in the endothelium. To substantiate the above hypothesis, we used proliferation assays, western blot analysis and quantitative fluorescence microscopy.

\section{Materials and methods}

\section{Cell culture}

Human umbilical arterial endothelial cells (HUAEC) (Cambrex Bio Science, Baltimore, MD) were maintained in EGM - 2MV Bulletkit (Cambrex BioScience), consisting of endothelial cell basal medium -2 and the following growth supplements: fetal bovine serum (FBS), hydrocortisone $(0.2 \mathrm{ml})$, human fibroblast growth factor-basic (hFGF-B) ( $2 \mathrm{ml})$, vascular endothelial growth factor (VEGF) $(0.5 \mathrm{ml})$, $\mathrm{R}^{3}$-insulin-like growth factor-1 ( $\left.\mathrm{R}^{3}-\mathrm{IGF}-1\right)(0.5 \mathrm{ml})$, ascorbic acid $(0.5 \mathrm{ml})$, human epidermal growth factor (hEGF) $(0.5 \mathrm{ml}), \mathrm{GA}-1000(0.5 \mathrm{ml})$. Cells were cultured in T-75 flasks at $37{ }^{\circ} \mathrm{C}$ in a $5 \% \mathrm{CO}_{2}$ atmosphere and they were split $1: 2$ twice a week.

\section{Proliferation assays}

HUAEC were plated at a density of 1000 cells/well in a 96-well plate. Cells were incubated with morphine, VEGF or SNAP (S-nitroso-N-acetylpenicillamine) in culture medium for three days. When using the inhibitor, cells were pretreated for four hours before adding morphine, VEGF, or SNAP.

Proliferation was measured by using the CellTiter - Glo ${ }^{\mathrm{TM}}$ Luminescent Cell Viability Assay (Promega, Madison, WI). It is a homogeneous method for determining the number of viable cells in a culture based on the quantification of the adenosine triphosphate content, which is proportional to the number of metabolically active cells. The luminescent signal, produced by the luciferase reaction is measured by a luminometer with an integration time of one second per well.

\section{Western blot analysis}

HUAEC cells were plated in petri-dishes at $\pm 600,000$ cells/ dish in complete culture medium. The next day, cells were treated for 30 minutes with morphine or VEGF and were then harvested into icecold lysis buffer (50 mM TRIS-HCl, $2 \mathrm{mM}$ EGTA, 1\% SDS and one tablet Complete Protease Inhibitor Cocktail [ORIGIN] per $50 \mathrm{ml}$ buffer). The different fractions were diluted 1:1 with sample buffer (Invitrogen, Carlsbad, CA) and boiled for four minutes. Equal volumes of the samples were subjected to gel electrophoresis in a $10 \%$ TRIS-glycine gel (Invitrogen) followed by transfer of the proteins to nitrocellulose membranes. Membranes were blocked with $0.1 \%$ Tween in PBS containing 10\% nonfat dry milk for 30 minutes at room temperature, incubated overnight at $4{ }^{\circ} \mathrm{C}$ either with p44/42 MAP Kinase-antibody (Cell Signaling, Beverly, MA) at a dilution of 1:100 or with phospho-p44/42 MAP-Kinase (Cell Signalling) at a dilution of 1:500 or with monoclonal anti-actin clone AC-40 (Sigma-Aldrich, St. Louis, MO) at a dilution of 1:200 and washed extensively. They were then incubated either with a horseradish peroxidase-conjugated anti-rabbit antibody at a dilution of 1:1000 or with a horseradish peroxidaseconjugated anti-mouse antibody at a dilution of 1:2000 for one hour at room temperature before being developed with the enhanced chemiluminescent substrate (SuperSignal ${ }^{\circledR}$ West Pico Chemiluminescent Substrate; Pierce, Rockford, IL).

\section{NO measurements}

For the real-time detection of NO, we used the NO indicator DAF-FM (4-amino-5-methylamino-2', 7'-difluoroscein) Diacetate (Molecular Probes, Eugene, OR), which is nonfluorescent until it reacts with NO to form a fluorescent benzotriazole. Cells were plated in a 96-well plate with a 
glass bottom, incubated with the diluted DAF-FM diacetate $(1 \mu \mathrm{M})$ for 30 minutes at $37^{\circ} \mathrm{C}$ and washed with buffer to remove excess probe. Cells were then incubated for an additional 30 minutes at room temperature to allow complete de-esterification of the intracellular probe. The image recording system consists of a Axiovert S135 inverted microscope (Zeiss, Oberkochen, Germany) equipped with a motorized (x,y)-stage (Märzhauser, Wetzlar, Germany), focus-drive and CCD-camera (Photonics, Shizuoka, Japan). Proprietary software allows automated focusing, image acquisition, and storage for each well. A 96 well plate is put on the stage and the user selects the fields containing cells to be imaged over time. When all desired fields are selected, images are recorded automatically during a preset time. The objects of interest were detected in each field and the fluorescence intensity of each object is continuously measured for 20 minutes (FITC filter). After minute 5, cells were stimulated with morphine or SNAP, a NO-donor (positive control). Each experiment was simultaneously performed with a control (vehicle alone) to exclude experimental drift in NO release unrelated to the study drugs.

\section{Statistical analysis}

For the proper analysis, the absolute values of each of the proliferation assays and NO measurements were converted into relative values, with the respective control condition set as $100 \%$. All of the data are expressed as mean \pm SEM. The data were evaluated with the nonparametric Mann-Whitney test using Minitab statistical software (Minitab Inc., State College, PA). Differences with the control group were considered statistically significant at $\mathrm{P}<0.05$.

\section{Results}

\section{Effects of morphine on endothelial cell proliferation}

To investigate if morphine has proliferative stimulus properties on endothelial cells, it was compared to VEGF. Proliferation assays were performed in the presence of different concentrations of the opioid $(10 \mu \mathrm{M}-10 \mathrm{nM})$, but also in the presence of VEGF and SNAP as positive controls. SNAP is a NO-donor under physiologic conditions. A significant stimulation of endothelial cell proliferation occurred in the range between $1 \mu \mathrm{M}$ to $10 \mathrm{nM}$ morphine $(\mathrm{P}<0.01$ versus control; Figure 1A). An increase in cell density of about $26 \%$ was measured with morphine $100 \mathrm{nM}$ and an increase of more than $30 \%$ with VEGF $100 \mathrm{ng} / \mathrm{ml}$. Also the release of NO by the NO-donor SNAP $50 \mu \mathrm{M}$ stimulated the proliferation of the HUAEC cells to a similar degree.
Effects of the MAPK-inhibitor PD98059 on endothelial cell proliferation

To illustrate the role of the MAPK pathway in mediating the morphine-induced proliferation, experiments were performed in the absence and the presence of the MAPK-inhibitor PD98059 (Figure 1B). Under every condition, there was a clear inhibition of the endothelial cell proliferation by the MAPK-inhibitor PD98059 at $3 \mu \mathrm{M}$, but the effect on the morphine dose range is larger than the effect on the control condition ( $\pm 25 \%$ decrease versus $12.14 \%$ decrease). As such, the MAPK - inhibitor PD98059 clearly inhibits the morphine - induced HUAEC proliferation.

\section{Effects of morphine on the NO production in HUAEC cells}

To evaluate the potential role of $\mathrm{NO}$ in the stimulation of HUAEC proliferation, NO production upon morphine addition was measured. A live cell imaging system was used to quantify intracellular NO levels at the single cell level by measuring the intensity of DAF-FM diacetate, a $\mathrm{NO}$-specific fluorescent dye. Experiments were done with different concentrations of morphine and SNAP as a positive control. Only the addition of SNAP $100 \mu \mathrm{M}$ resulted in an increase in fluorescent signal intensity which was statistically significant and different from the control condition $(\mathrm{P}<0.05$; Table 1).

Figure 2 illustrates the data sampling in the live cell imaging set-up. With the addition of SNAP $10 \mu \mathrm{M}$ no increase in the fluorescence signal was detected (Table 1), while $50 \mu \mathrm{M}$ was able to stimulate the proliferation of HUAEC (Figure 1A: 30\% increase in proliferation rate as compared with control cultures).

\section{Influence of morphine on the activation of MAPK}

Based on the results of the cell proliferation experiments with the MAPK-inhibitor, there was already an indication for a role for MAPK as mediator of the morphine induced HUAEC cell proliferation. To further substantiate this observation, the activation of the MAPK pathway was studied by western blots. The fraction of phosphorylated enzyme is indicative for the level of activation of the second messenger cascade and illustrates the effects of morphine on the MAPK activation cascade. An increased signal was observed with morphine and VEGF as demonstrated via detection with an antibody against the phosphorylated enzyme. Especially the highest morphine concentration 
A)

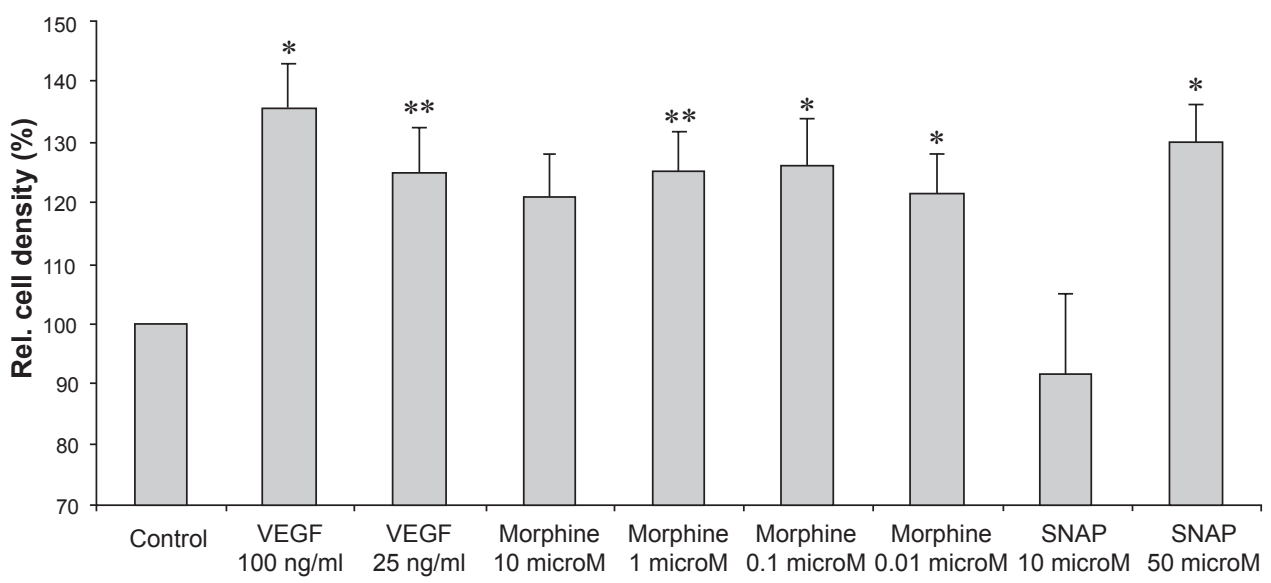

B)

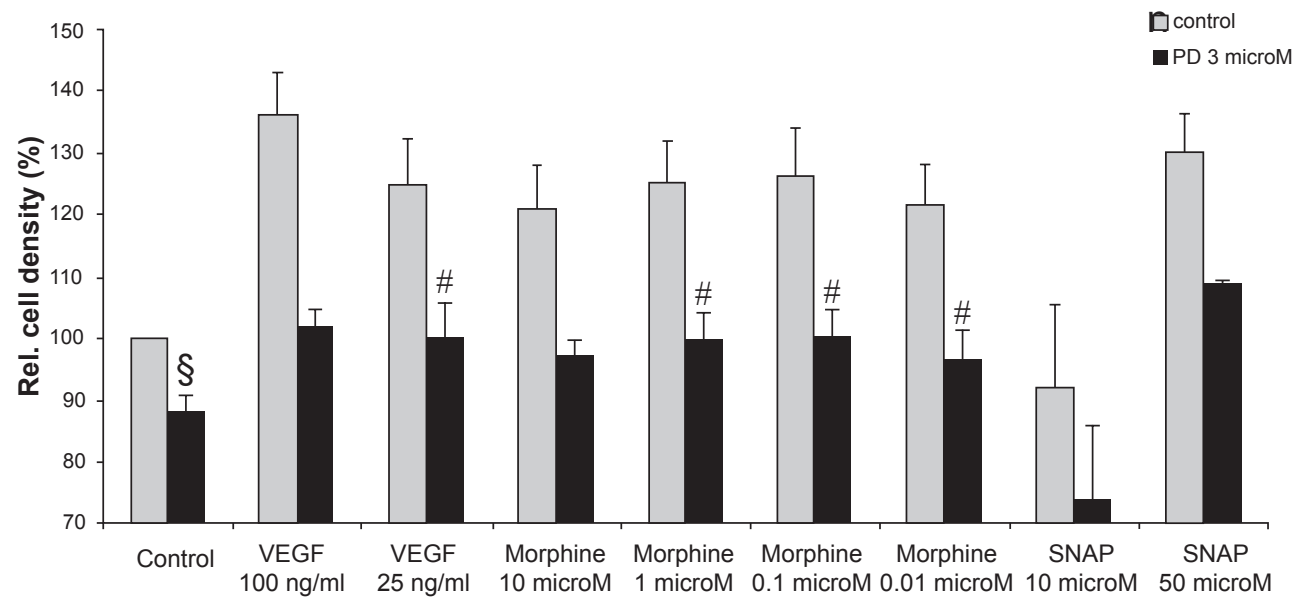

Figure I Morphine, VEGF, and SNAP $10 \mu$ M stimulate HUAEC proliferation after three days of incubation A).The MAPK-inhibitor PD98059 reduces the proliferation capacity of HUAEC cells after three days of incubation B).

Notes: Mann-Whitney U Test was used for statistical analyses; A) *P $<0.01$; **P $<0.005$, compared with control; B) ${ }^{*} \mathrm{P}<0.05$; $\$$ P $<0.005$, conditions with PD98059 compared with conditions without PD98059. Each experiment was repeated at least four times in triplicate $(\mathrm{n}=12)$, except PD/VEGF $100 \mathrm{ng} / \mathrm{ml}$ and PD/SNAP 50 and $10 \mu \mathrm{M}$, which were run twice $(n=8)$. Each value indicates mean; bars \pm SEM.

Abbreviations: HUAEC, human umbilical endothelial cells; MAPK, mitogen-activated protein kinases; SNAP, S-nitroso-N-acetylpenicillamine; VEGF, vascular endothelical growth factor.

$(10 \mu \mathrm{M})$ indicated a clear activation of MAPK (Figure 3 panel A top).

\section{Influence of PD98059 on the activation of MAPK}

As control for the use of the MAPK-inhibitor PD98059 in the proliferation assays, the effect of this inhibitor was also studied by western blotting. PD98059 inhibits the second enzyme (= MAPKK) in the cascade and as such inhibits the phosphorylation/activation of the next enzyme, namely the important MAPK. Treating HUAEC for 30 minutes with morphine or VEGF clearly induced the phosphorylation/activation of the MAPK enzyme (Figure 3, panel A). If we compare the samples without inhibitor with those with inhibitor (Figure 3, panel A versus panel B), a decrease of the signal was seen with the phospho-MAPK antibody (Figure 3, panel B at the top). Pre-treatment with $3 \mu \mathrm{M}$ PD98059 during four hours inhibited the morphine-induced MAPK phosphorylation. When using the total MAPK antibody, no decrease was observed (Figure 3, panel B in the middle). As a control a similar experiment was performed using an anti-actin antibody (Figure 3, panel B bottom). The signal was equal for all samples and indicative for the specificity of the MAPK-inhibitor assay.

\section{Discussion}

The potential effect of morphine on the proliferation of endothelial cells is extremely important merely because morphine is often used to treat pain all over the world without having well documented the effects on nonneuronal tissue. 
Table I NO quantification: overview of the fluorescencemeasurements per condition per phase. NO levels were measured by quantitative fluorescence microscopy in HUAEC cells exposed to buffer, SNAP (I00-10 $\mu \mathrm{M})$ or morphine (10 $\mu \mathrm{M}-10 \mathrm{nM})$. Cells were loaded with DAF-FM diacetate (I $\mu \mathrm{M})$ for 30 minutes before stimulation. Data are expressed as mean relative intensity \pm SEM, with the measurements in phase I (= before addition) set as 100\%

\begin{tabular}{|c|c|c|c|c|}
\hline \multicolumn{2}{|l|}{ Condition } & \multicolumn{2}{|c|}{$\begin{array}{l}\text { REL. Intensity + SEM } \\
\text { (P-value) }\end{array}$} & \multirow[t]{2}{*}{$\mathbf{N}$} \\
\hline Control & phase I & $100.00+0.00$ & $(/)$ & \\
\hline & phase 2 & $99.16+0.68$ & $(/)$ & 18 \\
\hline & phase 3 & $99.89+1.39$ & $(/)$ & \\
\hline SNAP & phase I & $100.00+0.00$ & $(/)$ & \\
\hline \multirow[t]{2}{*}{100 microM } & phase 2 & $|69.7|+47.23$ & $(p=0.0098)$ & 3 \\
\hline & phase 3 & $273.78+102.44$ & $(p=0.0129)$ & \\
\hline SNAP & phase I & $100.00+0.00$ & $(/)$ & \\
\hline \multirow[t]{2}{*}{10 microM } & phase 2 & $96.65+0.52$ & $(p=0.3085)$ & 2 \\
\hline & phase 3 & $94.44+0.87$ & $(p=0.0823)$ & \\
\hline Morphine & phase I & $100.00+0.00$ & $(/)$ & \\
\hline \multirow[t]{2}{*}{10 microM } & phase 2 & $99.68+1.26$ & $(p=0.6332)$ & 6 \\
\hline & phase 3 & $102.21+3.14$ & $(p=0.6332)$ & \\
\hline Morphine & phase I & $100.00+0.00$ & $(/)$ & \\
\hline \multirow[t]{2}{*}{ I microM } & phase 2 & $103.58+3.04$ & $(p=0.1039)$ & 3 \\
\hline & phase 3 & $103.70+3.47$ & $(p=0.1513)$ & \\
\hline Morphine & phase I & $100.00+0.00$ & $(/)$ & \\
\hline \multirow[t]{2}{*}{0.1 microM } & phase 2 & $99.78+0.60$ & $(p=0.3892)$ & 3 \\
\hline & phase 3 & $100.51+1.11$ & $(p=0.5030)$ & \\
\hline Morphine & phase I & $100.00+0.00$ & $(/)$ & \\
\hline \multirow[t]{2}{*}{0.01 microM } & phase 2 & $97.99+1.30$ & $(p=0.8574)$ & 2 \\
\hline & phase 3 & $98.06+1.26$ & $(p=0.6750)$ & \\
\hline
\end{tabular}

Abbreviations: DAF-FM, 4-amino-5-methylamino-2',7'-difluoroscein; HUAEC, human umbilical endothelial cells; No, nitric oxide; SNAP, s-nitroso-N-acetylpenicillamine.

Morphine induces pain relief by blocking the transmission of the sensory system. At first the effects of the opioids, especially on analgesic and anti-nociceptive properties, were examined. Later, a number of experiments have demonstrated that morphine modulates the activity of a variety of cell types, among them endothelial cells. Therefore, the interest in studies on the effects of opiates outside of the nervous system increased. ${ }^{5,8}$ For example, studies on the effects of morphine on tumor growth were performed. ${ }^{9}$ After all, angiogenesis is often associated with the pathogenesis of tumor progression and metastasis. Despite the widespread use of opioids to treat pain in patients with cancer, little is known about the effects of these drugs on vascular endothelium, a key player in angiogenesis, and hence tumor growth.

Angiogenesis is a complex process regulated by anti- and pro-angiogenic factors. One of these factors specific for the endothelium is VEGF. In this study, we compared the opiate morphine with the positive control VEGF. The biological effects of both are mediated by two different receptors.
Morphine acts on the $\mu$-opioid receptor (GPCR-type), while VEGF acts on tyrosine kinase receptors, which are expressed mainly on the vascular endothelium. . $^{5,10}$

By using the proliferation assays, we were able to illustrate an increased cell number after treatment with morphine. The effects of morphine approximate the mitogenic and pro-survival activities of VEGF. Morphine induced a nice dose-dependent effect between $10 \mu \mathrm{M}$ and $10 \mathrm{nM}$. Morphine is used clinically in doses of $10-2450 \mathrm{mg} /$ day, resulting in serum concentrations that are between $2 \mathrm{nM}$ to $3.5 \mu \mathrm{M} .{ }^{9}$ We found a significant proliferative stimulus with morphine in the range of $1 \mu \mathrm{M}$ to $10 \mathrm{nM}(\mathrm{P}<0.05$ versus control). Thus, at clinically relevant concentrations morphine stimulates endothelial cell proliferation.

The association of the tyrosine kinase receptor with downstream activation of the MAPK pathway is common knowledge and the fact that VEGF has an effect on endothelial cells through the MAPK pathway was shown. ${ }^{10,11}$ Besides this, it is also known that the activation of the MAPK-cascade can be the consequence of the interaction between a GPCR, like MOR, and its ligand, in our case morphine., ${ }^{412}$ This signaling pathway is one of the most important cascades that convert an extracellullar signal into an intracellular response. The regulation of genes by this way regulates biological processes among which proliferation. ${ }^{6}$ Hence, we investigate the importance of MAPK within the morphine stimulus. Dissection of the intracellular pathway giving rise to the effect and possible side-effects is after all important for therapeutic applications. First, we checked the involvement of the MAPK-cascade by using an inhibitor, PD98059, which works particularly on the second enzyme. In this way, the final enzyme will not be activated and not be able to regulate gene expression in the nucleus. The results of the proliferation experiments with and without inhibitor PD98059 in VEGF- and morphine-treated cells illustrated a decrease in cell number of $25 \%$ and $13 \%$ after pretreatment, respectively. This underlines the role of the MAPK-pathway in the VEGFand morphine-induced proliferative stimulus.

We also looked at the activation of the MAPK pathway by using western blot analysis. Both VEGF and morphine $10 \mu \mathrm{M}$ induced a significant increase in phosphorylated MAPK when compared to the controls. This indicates an activation stimulus by both factors on the MAPKcascade. It is striking that, in the proliferation experiments, a maximal stimulus was seen with morphine $100 \mathrm{nM}$, while the activation of MAPK was the strongest with morphine $10 \mu \mathrm{M}$. This can point to differences in exposure time; only 30 minutes for the western blot experiments while the proliferation experiments were quantified after chronic exposures. 


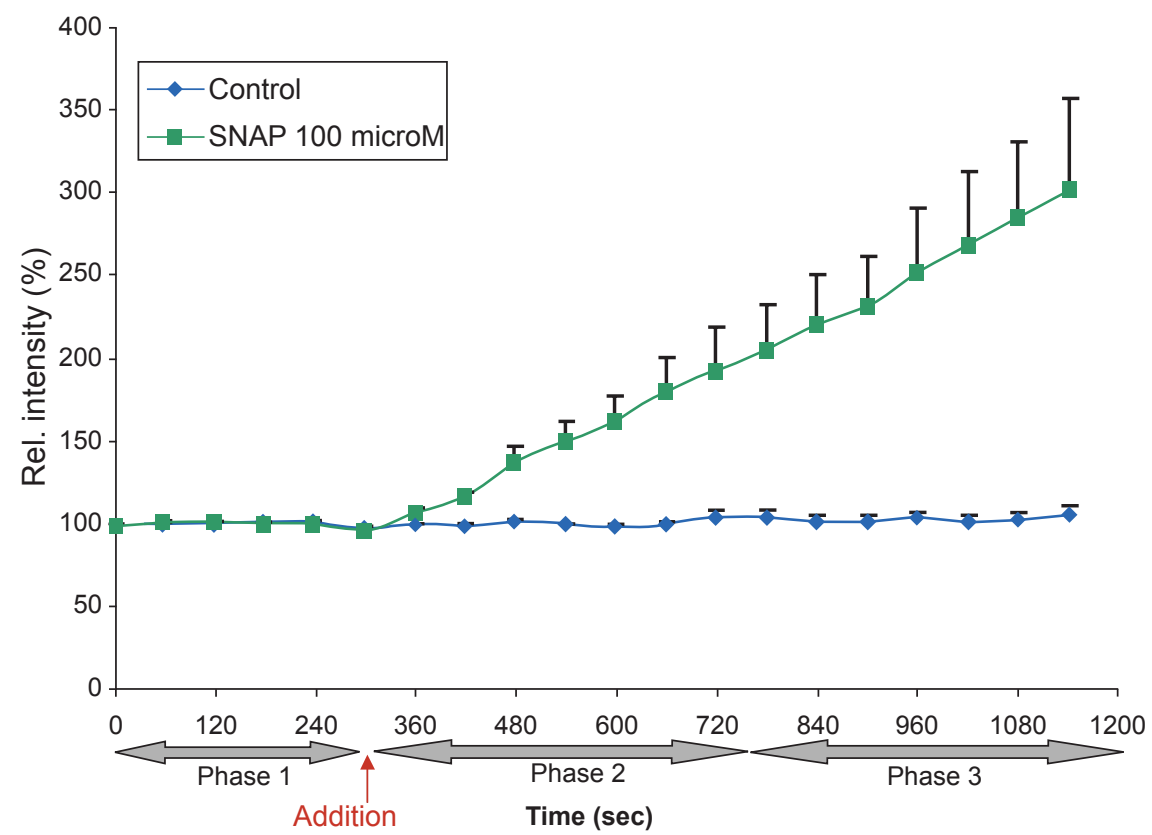

Figure 2 NO quantification: effect of the addition of SNAP $100 \mu \mathrm{M}$ with regard to the control (addition of buffer) in function of the time. To illustrate the processing of the data, the results of one experiment are presented. HUAEC were exposed to SNAP $100 \mu M$ (the positive control) or vehicle (control). The relative intensity, measured in every cycle is plotted against time (one measurement per minute). After cycle/minute 4, the drug was added while the measurement continued. For the finale analysis, the values are grouped, as pointed out in the graph: phase I, phase 2 and phase 3.All the measurements in phase I are thus control measurements before the addition of the compound of interest.

Abbreviations: HUAEC, human umbilical endothelial cells; NO, nitric oxide; SNAP, s-nitroso-N-acetylpenicillamine.

We mainly focused on the proliferation of endothelial cells, but the MAPK cascade can also be involved in other biological processes, like differentiation and apoptosis. ${ }^{13,14}$ Besides this, the phenomenon of 'desensitization' can also contribute to this fact. It is known that a continuous stimulation of the GPCR can lead to a decrease in response or briefly, "desensitization". ${ }^{15,16}$ In our study of the activation of the MAPK pathway, we observed the highest signal with morphine $10 \mu \mathrm{M}$.

Also the specificity of the MAPK pathway was shown by the use of the inhibitor PD98059 and confirmed by western blot. We showed that the inhibitor was able to inhibit the

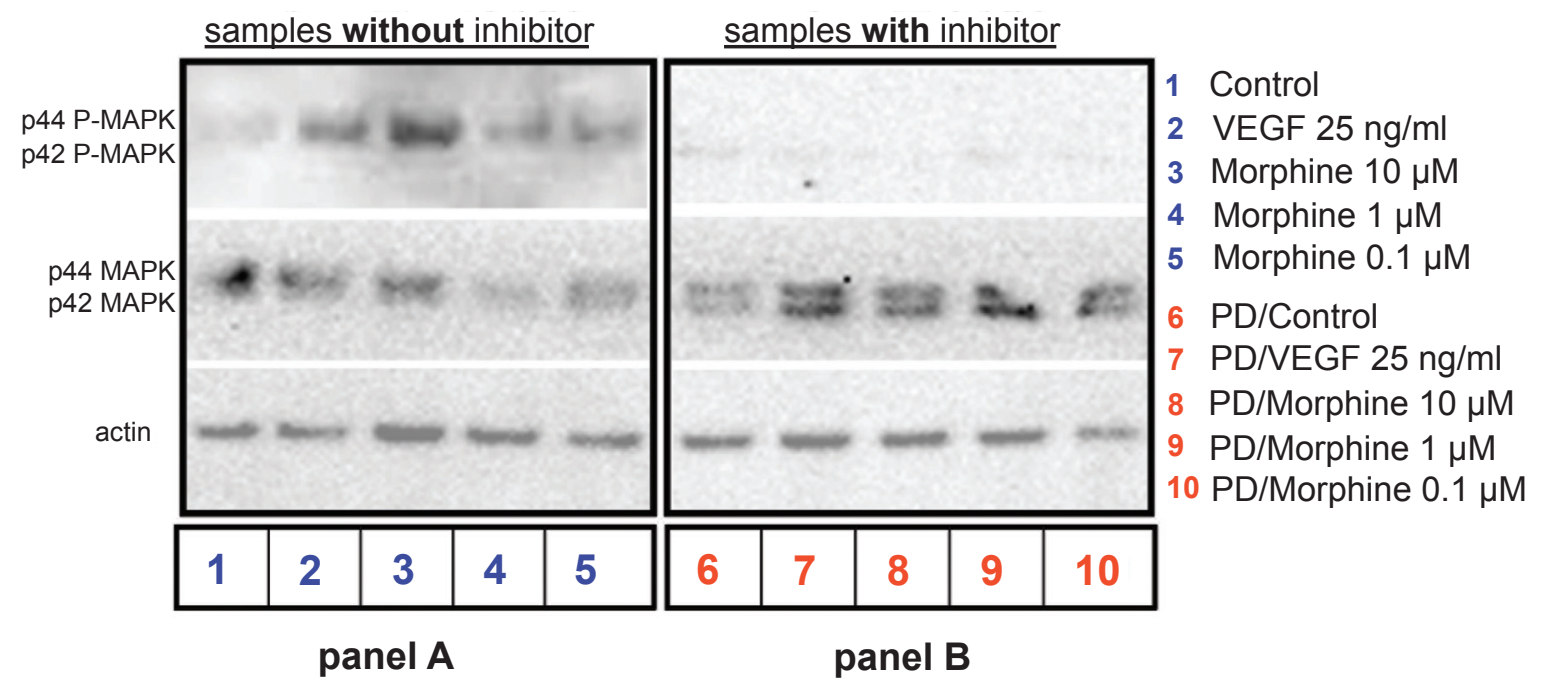

Figure 3 A) Effect of morphine on the activation of MAPK. Treatment during 30 minutes with morphine orVEGF induced the phosphorylation/activation of the MAPK enzyme. B) Effect of the MAPK-inhibitor PD98059 on the activation of MAPK. Pretreatment with $3 \mu$ M PD 98059 during four hours inhibited the morphine-induced MAPK phosphorylation; no changes in the total MAPK and actin signal were seen. Control indicates unstimulated cells. The actine label was performed by re-probing the MAPK membranes. Abbreviations: MAPK, mitogen-activated protein kinases; VEGF, vascular endothelical growth factor. 
proliferative stimulus of morphine and this provides further support for the hypothesis that MAPK plays an essential role in the proliferation behavior of the endothelial cells induced by morphine.

In studies on the characterization of the cellular pathways involved in the VEGF-induced proliferation of endothelial cells, it was already clear that the mitogenic activity of VEGF requires the activation of the MAPK cascade. Besides this, it was also known that NO/cGMP contributed to the pro-angiogenic properties of vascular endothelial growth factor in human endothelial cells. ${ }^{7,17}$ Hence, we investigated also the role of NO to the underlying mechanisms of morphine. However, we were not able to detect a significant increase of the amount NO by using quantitative fluorescence, despite the positive results with the NO donor SNAP $100 \mu \mathrm{M}$. The only indication for a possible role of NO within the morphine-induced proliferative stimulus, were the results of the cell density measurements: in our experimental approach, we made use of the NO-donor SNAP. The increase in cell density induced by SNAP $50 \mu \mathrm{M}$ approximated the increased proliferation by VEGF $(30 \%)$. These results only indicate that NO stimulates the mitogenic activity of HUAEC cells. The question if morphine is able to stimulate the NO production remains unclear after our study.

For future experiments, it is important to further clarify the role of $\mathrm{NO}$ by performing proliferation experiments in the presence of a NO inhibitor (eg, L-NAME). The results will indicate if $\mathrm{NO}$ is required for the morphine-induced proliferation.

In a second part, one can compare the effect of morphine with other pain-relieving drugs. Because the clinically used opioids consist of different chemical classes of compounds having different binding and functional profiles at opiate and nonopiate receptors, it is theoretically possible that the effects observed with morphine can not be generalized to all other agents. By doing the same experiments with other agents, one will be able to detect any difference in response.

Furthermore, because of the many different phenotypes of tumors, some differentiation might be possible and as a consequence the effects of various opioids in various cancer cell lines should be evaluated before making any generalized conclusions.

It is also very important to find out if the increase in proliferation, induced by morphine, can actually give rise to a higher pro-angiogenic activity in animal models. It is not certain that the increase in cell density of about $26 \%$, seen in these in vitro experiments and at high morphine concentrations, also results in an increased activity of the blood vessel forming process in in vivo experiments. Therefore, it is necessary to evaluate the effect of morphine on the growth and angiogenesis in tumors of different phenotype. The evaluation can be done via histological quantification of the tumor vascularisation and via $\mu \mathrm{CT}$. Our working hypothesis can then be confirmed or contradicted by the results of those in vivo experiments.

Finally, we can conclude that the pro-angiogenic activity of morphine might have implications for the therapeutic use of this opiate in a clinical setting if additional and especially in vivo experiments confirm the present observations. The stimulating effect on the proliferation of endothelial cells seems a breakthrough in cardiovascular medicine and wound healing. In contrast, morphine administration to patients with cancer might inadvertently increase angiogenesis, raising concerns about the widespread use of this analgesic in those patients if the in vitro results can be translated to the in vivo situation in humans. It is therefore important to indicate that the in vitro data obtained can not simply be generalized to humans or to other analgesics.

\section{Disclosure}

The authors report no conflicts of interest in this work.

\section{References}

1. Carmeliet P, Jain RK. Angiogenesis in cancer and other diseases. Nature. 2000;407:249-257.

2. Rosen LS. Clinical experience with angiogenesis signaling inhibitors: focus on vascular endothelial growth factor (VEGF) blockers. Cancer Control. 2002;9:36-44.

3. Schmidt H, Kirkpatrick J, Kamp G, Wegener G, Peters K. Angiogenesis as a pharmalogical target. G.I.T. Laboratory Journal. 2001;5.

4. Zhang J, Ferguson SS, Barak LS, et al. Role for G protein-coupled receptor kinase in agonist-specific regulation of mu-opioid receptor responsiveness. Proc Natl Acad Sci US A. 1998;95:7157-7162.

5. Stefano GB, Hartman A, Bilfinger TV, et al. Presence of the mu3 opiate receptor in endothelial cells. Coupling to nitric oxide production and vasodilation. J Biol Chem. 1995;270:30290-30293.

6. Kolch W. Meaningful relationships: the regulation of the Ras/Raf/MEK ERK pathway by protein interactions. Biochem J. 2000;351:289-305.

7. Parenti A, Morbidelli L, Cui X-L, et al. Nitric oxide is an upstream signal of vascular endothelial growth factor-induced extracellular signal-regulated kinase $1 / 2$ activation in postcapillary endothelium. J Biol Biochem. 1998;273:4220-4226.

8. Dobrenis K, Makman MH, Stefano GB. Occurrence of the opiate alkaloid - selective $\mu_{3}$ receptor in mammalian microglia, astrocytes and Kupffer cells. Brain Res. 1995;686:239-248.

9. Gupta K, Kshirsagar S, Chang L, et al. Morphine stimulates angiogenesis by activating proangiogenic and survival-promoting signaling and promotes breast tumor growth. Cancer Res. 2002;62:4491-4498.

10. Yu Y, Sato JD. MAP kinases, phosphatidylinositol 3-kinase, and p70 S6 kinase mediate the mitogenic response of human endothelial cells to vascular endothelial growth factor. J Cell Physiol. 1999;178. $235-246$. 
11. Gille H, Kowalski J, Li B, et al. Analysis of biological effects and signaling properties of Flt-1 (VEGFR-1) and KDR (VEGFR-2). A reassessment using novel receptor-specific vascular endothelial growth factor mutants. J Biol Chem. 2001;276:3222-3230.

12. Schmidt H, Schulz S, Klutzny M, Koch T, Händel M, Höllt V. Involvement of mitogen-activated protein kinase in agonist-induced phosphorylation of the mu-opioid receptor in HEK 293 cells. J Neurochem. 2000;74:414-422.

13. Lodish H, Baltimore D, Berk A, Zipursky SL, Matsudaira P, Darnell J. Molecular Cell Biology. 4th Edition. New York, NY: W. H. Freeman and Company; 2000.
14. Cobb, 1996.

15. Harrison LM, Kastin AJ, Zadina JE. Opiate tolerance and dependence: Receptors, G-proteins, and antiopiates. Peptides. 1998;19:1603-1630.

16. Law P-Y, Wong YH, Loh HH. Molecular mechanisms and regulation of opioid receptor signaling. Annu Rev Pharmacol Toxicol. 2000;40:389-430.

17. Papapetropoulos A, Garcia-Cardeña G, Madri JA, Sessa WC. Nitric oxide production contributes to the angiogenic properties of vascular endothelial growth factor in human endothelial cells. J Clin Invest. 1997;100:3131-3139. 